\title{
Article
}

\section{What is the impact of physical effort on the diagnosis of concussion?}

Brauge, D., Dechambre, X., Carling, Christopher, Decq, P., Pillard, F., Mrozek, S., Piscione, J., and Yrondi, A.

Available at http://clok.uclan.ac.uk/28013/

Brauge, D., Dechambre, $X$., Carling, Christopher ORCID: 0000-0002-74563493, Decq, P., Pillard, F., Mrozek, S., Piscione, J., and Yrondi, A. (2019) What is the impact of physical effort on the diagnosis of concussion? Clinical Journal of Sport Medicine. ISSN 1050-642X

It is advisable to refer to the publisher's version if you intend to cite from the work.

For more information about UCLan's research in this area go to

http://www.uclan.ac.uk/researchgroups/ and search for <name of research Group>.

For information about Research generally at UCLan please go to http://www.uclan.ac.uk/research/

All outputs in CLoK are protected by Intellectual Property Rights law, including Copyright law. Copyright, IPR and Moral Rights for the works on this site are retained by the individual authors and/or other copyright owners. Terms and conditions for use of this material are defined in the policies page. 


\section{What is the impact of physical effort on the diagnosis of concussion?}

David Brauge MD, Xavier Dechambre, MD, Christopher Carling, PhD, Philippe Decq, MD, Fabien Pillard, PD, Segolène Mrozek, MD, PhD, Julien Piscione, PhD, Antoine Yrondi, MD

David Brauge, MD: Department of Neurosurgery, University Hospital of Toulouse, Toulouse, France

Xavier Dechambre, MD: Depatment of General Medicine, University Hospital of Toulouse, Toulouse, France

Christopher Carling, PhD: Institute of Coaching and Performance, University of Central Lancashire, Preston, UK

Fabien Pillard MD PhD: Department of Exploration of Respiratory Function and Sports Medicine, University Hospital of Toulouse, Toulouse, France

Philippe Decq, MD PhD: Department of Neurosurgery, Assistance Publique des Hôpitaux de Paris, University Hospital of Beaujon, Clichy, France

Mrozek Segolene, MD PhD: Department of Anesthesioloy and Intensive Care, University Hospital of Toulouse, Toulouse, France

Julien Piscione, PhD: Research Department, French Rugby Union Federation, Marcoussis, France

Antoine Yrondi, MD, PhD: Department of Psychiatry, University Hospital of Toulouse, Toulouse, France

Corresponding Author:

Dr David Brauge, Pôle Neuroscience - Neurochirurgie, Hôpital Purpan, 31059 Toulouse -

France. (brauge.d@ chu-toulouse.fr). Tel.: +33561779510. Fax: +33561322240 


\begin{abstract}
:
Objective:

Sport-related concussion commonly occurs in contact sports such as rugby. To date, diagnosis is based on the realization of clinical tests conducted pitch-side. Yet, the potential effect of prior physical effort on the results of these tests remains poorly understood. The purpose of this study was to determine whether preceding physical effort can influence the outcome of concussion assessments.
\end{abstract}

\title{
Design:
}

Prospective observational study.

\section{Setting:}

University Medicine Center

\section{Patients:}

A cohort of 40 subjects ( 20 rugby players and 20 athletes from a range of sports).

\section{Intervention:}

A concussion assessment was performed immediately following physical activity. Following a period of 6 months and under the same experimental conditions, the same cohort performed the same tests in resting conditions.

\section{Main outcome measure:}

Results of concussion tests.

\section{Results:}

In both cohorts, the comparison for post-exercise and rest assessments demonstrated a most likely moderate-to-very large increase in the number of symptoms, severity of symptoms and BESS score. In the rugby cohort, scores for concentration, delayed memory and SAC, likelyto-most likely decreased following completion of physical activity compared to baseline values. The between-cohort comparison showed a greater impact post-exercise in the rugby players for delayed recall $(0.73 \pm 0.61,93 / 7 / 1)$ and SAC score $(0.75 \pm 0.41,98 / 2 / 0)$.

\section{Conclusion:}

Physical activity altered the results of concussion diagnostic tests in athletes from a range of sports and notably in rugby players. Therefore, physical efforts prior to the concussion incident should be accounted for during pitch-side assessments and particularly during rugby competition and training.

Keywords: Concussion, physical exertion, SCAT, HIA, Rugby 


\section{Introduction:}

Concussion is a traumatic condition that has received much attention in the medical literature in recent years and particularly in the sport of rugby (1). It is defined as a "trauma resulting in the rapid onset of short-lived impairment of neurological function that resolves spontaneously" (2). Signs of concussion observed in the acute phase indicate a brain dysfunction that in general recovers spontaneously within a few days. However, traumas can occur repeatedly over the course of a player's career. The occurrence of a second concussive event within a short time period can lead to Second Impact Syndrome (SIS) which is due to incomplete recovery following the first concussion. Despite occurring rarely, SIS can prove fatal (3). Over a longer period, the accumulation of concussion incidents might lead to disabling pathologies such as prolonged post-concussive syndrome $(4,5)$ and/or neurodegenerative disorders $(6)$.

These concerns have prompted international sports governing bodies to adopt guidelines based on expert-based consensus (2). These expert recommendations firstly emphasize the importance of not disregarding the risk that a concussive event might have occurred whilst proposing a battery of clinical tests to aid pitch-side diagnosis immediately following an event. These procedures aim to reduce the risk that a player who is concussed continues to compete. This battery of tests, known as the Sport Concussion Assessment Tool (SCAT) was proposed in its first version in 2005 (7). In the sport of rugby, the protocol is broken down into a threestage diagnostic process known as the Head Injury Assessment (HIA) (8). According to Raftery: "this three-stage process was introduced recognising that concussion has evolving symptoms". If a concussion event is confirmed pitch-side using the HIA 1st stage (HIA1), the player is immediately and permanently removed from play. In the case of normal HIA1, the athlete is allowed to go back to the field but two additional assessments are required: 3 hours after the accident (HIA2) and at 48 hours (HIA3). In an international rugby tournament 18,3\% of concussion were diagnosed only by this delayed assessment (ref Fuller).

The HIA1 is a 10 min off-field assessment tool employed when a player has possibly incurred a head injury and for which the immediate diagnosis is unclear (9). The assessment includes several tests from the SCAT battery (Table 1). The results derived from these tests are compared with those from a pre-season baseline assessment conducted away from any concussion episode. Since the publication of the first concussion-specific diagnosis tool proposed by McCrea $(10,11)$, it is recommended that this baseline assessment is performed immediately following physical effort in order to imitate in-competition conditions occurring prior to assessment. However, to our knowledge, there is no study that has investigated the potential impact of prior physical exertion on the entire HIA1 diagnostic protocol.

The main objective of this study is to determine if results derived from the HIA1 assessment are affected by physical exertion outside of any concussion event in both rugby players and other sports participants. Secondary objectives are to 1) evaluate the effects of physical fatigue on the entire SCAT diagnostic battery and 2) determine whether differences exist in the results of these tests between a high-risk group (rugby players) and athletes from other sports.

\section{Methods:}

Design: 
This prospective observational study investigated the effects of physical exertion on the diagnosis of concussion in a cohort of male athletes from a range of sports. The sample population included 40 athletes who were assessed individually on two occasions: 1) participants performed diagnostic concussion tests immediately following exercise commonly involving strenuous physical effort, 2) the cohort then performed the same tests in resting conditions outside of any physical activities. This second assessment was conducted 6 months after the initial one under the same experimental conditions (time and location). For each clinical test the post-exercise and resting values were compared.

\section{Inclusion and exclusion criteria:}

The cohort included male athletes between the ages of 20 and 40 inclusive (mean age: $31.5 \mathrm{SD}$ 4.1 years). Athletes were divided into two groups according to their sport: group 1 (rugby) consisting of 20 amateur rugby players (national standard) and group 2 consisting of 20 participants (other sports) regularly performing sports activities (at least 3 times per week) including track and field, cycling or cross-fit. Exclusion criteria included: presence of neurological, vestibular or psychiatric pathologies and diagnosis of a concussion event either in the previous 6 months prior to or during the study. The number to obtain a necessary sample size was calculated a priori using the central limit theorem.

\section{Analysis criteria:}

Each participant underwent a full evaluation including the diagnostic tests of the HIA battery. This included the following evaluations conducted in the following order:

- Maddock score:

- Immediate memory (5 words):

- Concentration test (digits backward and months in reverse order)

- Tandem walk (walk heel-to-toe down a 3-metre line and back)

- Symptoms score (from a list of 22 symptoms at the time of the test)

- Delayed recall (5 words)

The sum of the recall test results (immediate and delayed) and concentration test provided the Standardized Assessment of Concussion (SAC) score (10).

The modified version of the Maddock score was used for the other sport athletes (12). This test was not repeated during the rest assessment.

Additional tests in the SCAT battery were also used:

- Severity of symptoms experienced (scale 0 to 6 )

- Temporal orientation

- Balance test using the Balance Error Scoring System (BESS)

- Finger-nose test

\section{Method:}

The concussion diagnostic tests were firstly conducted immediately after a sustained physical effort of at least 30 minutes duration: pitch-side at the end of match-play for the rugby group, or immediately in the changing room following cessation of activity for the other sports athletes. 
In all cases this delay was less than 5 minutes respecting the HIA protocol adopted by World Rugby (9). All tests were performed by the same physician, trained in the management of concussion episodes. At the end of the first evaluation, if no concussion was determined, the athlete was allowed to participate in the second part of the study. The second evaluation was conducted, six months later, under resting conditions with a minimum of 24 hours interval respected after the last sporting activity. The tests were conducted by the same investigator, under comparable conditions, notably for the order of the clinical tests. In order to limit test / retest learning the list of words to be recalled immediately and delayed was modified between the two parts of the study.

\section{Statistical analysis:}

Data in text and figures are presented as means with standard deviations (SD) and 90\% confidence limits/intervals (CL/CI). Differences between rest and post-exercise results in the different variables, as-well-as between-group (rugby versus other athletes) differences in the changes, were examined using standardised differences (ES), based on Cohen's effect size principle. Probabilities were used to make a qualitative probabilistic mechanistic inference about the true changes/differences in the changes, which were assessed in comparison to the smallest worthwhile change $(0.2 \mathrm{x}$ pooled SDs $)(13,14)$. The scale was as follows: $25-75 \%$, possible; 75-95\%, likely; 95-99\%, very likely; $>99 \%$, almost certain. Threshold values for standardized differences were $>0.2$ (small), $>0.6$ (moderate), $>1.2$ (large) and very large $(>2)$.

\section{Ethical consideration:}

All participating athletes were explained the study requirements and signed a consent form between 01 November 2016 and 31 January 2017. This study was endorsed by the local ethics committee.

\section{Results:}

The final analysis included 37 athletes; 2 did not continue participation and 1 was excluded following a concussion episode sustained in the second part of the study. Physical and clinical characteristics of the two groups are presented in Table 2. Overall, at rest, there were no clear clinical differences between the two groups. Rugby players presented a very likely moderately lower concentration score than the other athletes $(3.5 \pm 0.9$ vs $4.1 \pm 0.6,-19.6 \pm 15.4 \%)$ but a likely slightly higher recall memory score $(4.1 \pm 0.9$ vs $3.6 \pm 1.2,+16.1 \pm 15.5 \%)$. The immediate memory score was likely slightly different between the two groups but this was not clinically substantial $(14.8 \pm 0.4$ vs $15 \pm 0)$.

\section{Within-group differences between baseline and post-effort values}

Differences between baseline values and post effort in the different tests are presented in figure $\mathbf{1}$ for the two groups (rugby and other athletes). Symptoms encountered after efforts are listed in the table 3 for both groups. There was no clear difference between post effort orientation score or immediate memory score in both groups. In the rugby group, the score for concentration $(3.5 \pm 0.9$ vs. $3.1 \pm 0.9$, ES $=-0.44 \pm 0.43$, \% chances: $1 / 17 / 82)$, delayed memory $(4.1 \pm 0.9$ vs. $3.7 \pm 1.2,-0.40 \pm 0.45,2 / 21 / 78)$ and SAC, $(27.4 \pm 1.7$ vs. $26.3 \pm 1.5,-0.61 \pm 0.23$, 0/0/100) likely-to-most likely decreased following completion of physical activity compared with baseline values. In the rugby group, there was also a most likely moderate-to-very large increase in the number of symptoms $(0.1 \pm 0.2$ vs. $2.1 \pm 1.6,1.69 \pm 0.54,100 / 0 / 0)$, severity of 
symptoms $(0.1 \pm 0.2$ vs. $3.5 \pm 2.8,1.68 \pm 0.53,100 / 0 / 0)$ and BESS score $(1.7 \pm 1.6$ vs. $3.3 \pm 2.2$, $0.76 \pm 0.30,100 / 0 / 0)$ following physical effort compared to baseline values.

In the other sports group, the concentration score was likely moderately decreased $(4.1 \pm 0.6 \mathrm{vs}$ $3.7 \pm 1.0$, ES $=-0.82 \pm 0.83$, \% chances: $2 / 8 / 90$ ) while the recall memory score likely slightly increased (3.6 \pm 1.2 vs. $3.9 \pm 1.0,0.33 \pm 0.41,71 / 27 / 2)$ following physical effort compared to baseline values. No clear differences were observed between SAC score post-effort versus baseline values. In the other sports group, there was also a most likely moderate-to-very large increase following physical effort in the number of symptoms $(0.1 \pm 0.5$ vs. $2.6 \pm 2.3,1.39 \pm 0.54$, $100 / 0 / 0)$, severity of symptoms $(0.1 \pm 0.5$ vs $3.8 \pm 3.6,1.38 \pm 0.53,100 / 0 / 0)$ and BESS score $(1.5 \pm 1.2$ vs. $2.7 \pm 1.9,0.72 \pm 0.30,99 / 1 / 0)$ compared to baseline values.

\section{Between-group differences in the changes between baseline and post-effort values}

Between-group (rugby vs other sports) differences in the changes between baseline and posteffort values are presented in table 2 and Figure 2. There was no clear difference between changes in post-effort versus baseline values in scores for immediate memory, concentration, symptoms, severity of symptoms and SAC between the rugby and other sports groups. Differences between baseline and post-effort in orientation scores were possibly slightly higher in the rugby compared to the other sports group ( $\mathrm{ES}=0.26 \pm 0.45,59 / 37 / 5)$. Recall memory score changes were likely largely higher $(0.73 \pm 0.61,93 / 7 / 1)$ and changes in SAC score were very likely moderately higher $(0.75 \pm 0.41,98 / 2 / 0)$ in the rugby versus the other sports group.

\section{Discussion:}

The present results suggest modifications in the outcome of several concussion diagnostic tests when associated with significant physical exertion. This change occurred for the concentration tests, delayed recall, SAC score and BESS equilibrium break number, number of reported symptoms and their severity score. The present findings tend to confirm those reported by McCrea (11) who first proposed physical stress tests to account for the effects of physical exertion on diagnosing concussion events.

A study by Lee et al (15) investigated the effects of fatigue on 82 professional athletes in whom the SCAT 3 protocol was applied. Their study showed that physical exertion caused an increase in the number of symptoms and errors in the balance test. Unlike the present report however, no alteration in performance in the cognitive tests was observed. Two criticisms can be directed at Lee's study: first, the two assessments (rest and post-effort) were performed at 3-weeks intervals thus there was a possible learning effect. Second, the post-exercise test conducted following 5 minutes of cycling at $75 \%$ of the participants' maximum heart rate might not be representative of the type of physical effort encountered when assessing potentially concussed athletes pitch side, particularly in rugby players (16). Other studies evaluating the impact of an aerobic type physical effort on balance tests specifically have reported a clear post-exercise alteration $(17,18,19)$, whereas moderate intensity exercise seemed to improve performance (20). The manifestation of concussion symptoms following intense physical exertion has led to one of the notable changes in the latest version of the SCAT; symptoms must be examined in a state of rest several minutes after leaving the field of play (2). 
The SCAT battery has been available since 2004 (21) and is regularly revised to ensure improvements in its diagnostic capacity $(22,23)$. While normative reference values are now available to help judge the results of the SCAT in top athletes (24), gold standard values are derived from comparisons of results against benchmark values recorded at the beginning of the season. The present findings confirm that this individual baseline assessment must be conducted under conditions that are highly similar to the athletes' habitual sporting activities. It is noteworthy that previous publications evaluating performance in baseline assessments frequently do not report the methodologies used to perform these fatigue protocols. This discrepancy may explain inconsistencies regarding their sensitivity and specificity (25). As regards the HIA protocol, initial assessments are also based on video observations that are useful in identifying concussion although identification is dependent upon observer experience $(26,27)$. The 3-step evaluation process to diagnose (or rule out) concussion is of note as it accounts for the evolution of symptoms (8). However, World Rugby regulations allow a player to leave the field of play for 10 minutes to perform a concussion test (HIA1), whereas the 2017 Berlin recommendations advise execution of the test following 10 minutes of rest (2). To our knowledge, the diagnostic performance of the full HIA protocol has not been evaluated.

\section{Difference between rugby vs other sports participants:}

Here, SAC changes were more substantial following rugby participation compared to the other sports. A closer analysis of the results reveals that the values for the "delayed recall" variable were impacted more in the rugby players. This suggests that the impact of physical exertion on concussion diagnostic tests was dependent upon sports activity and potentially its physical demands. This finding also raises the question regarding the potential role of repeated nonconcussive (non-concussion impact) head injuries on cognitive performance in rugby players immediately following rugby play $(28,29,30)$. The design of our study does not allow us to formulate conclusions on this issue and thus to this effect research is warranted. Finally, the latest version of the HIA protocol adopted by World Rugby indicates that the baseline assessment is performed after a sustained effort of 10 minutes. However, our results suggest that the results obtained are not comparable to those obtained following a rugby match.

\section{Limitations and bias}

The latest version of the SCAT (SCAT 5) was published after the start of the current study (2). However, the latter was conducted using assessments from the previous version which are very similar to those in the SCAT 5 since the only modification concerns the immediate memory and delayed recall parts in order to avoid a potential ceiling effect. To minimize the "practice effect" we modified the lists of words and numbers presented, as well as observing a period of 6 months between the two test phases. This duration is longer than the time needed to clear this bias which is habitually about 1 week (22). Further potential limitations were that the current study population included amateur male athletes with a relatively high mean age: $31.53 \pm 4.10$ years and participated in 3 training sessions per week. It is reasonable to suggest that the impact of physical exertion on the present test results might be associated to a better recovery capacity in younger and/or professional athletes. Extending this work to a larger population including participants of different ages, gender and practice levels would provide a more comprehensive analysis of the potential influence of fatigue in relation to these parameters. 


\section{Conclusion:}

This work demonstrates the effect of physical exertion on the results from HIA and SCAT concussion diagnostic assessments. Findings indicate that a baseline assessment should be conducted as frequently as possible following a physical effort resembling that of the sport activity in question in order to facilitate analysis of potential concussion events pitch side. Results also confirm that any search for symptoms following a potential event must be performed following a rest period of several minutes duration due to the effects of prior physical effort. 


\section{References:}

1:Prien A, Grafe A, Rössler R, et al. Epidemiology of Head Injuries Focusing on Concussions in Team Contact Sports: A Systematic Review. Sports Med. 2018;48(4):1-17.

2:McCrory P, Meeuwisse W, Dvorak J, et al. Consensus statement on concussion in sport- the 5th international conference on concussion in sport held in Berlin, October 2016. Br J Sports Med. 2017; 51(11):838-847.

3:Weinstein E, Turner M, Kuzma BB, et al. Second impact syndrome in football: new imaging and insights into a rare and devastating condition: case report. J Neurosurg Pediatr. 2013; 11(3):331-334.

4:Eisenberg MA, Andrea J, Meehan W, et al. Time interval between concussions and symptom duration. Pediatrics. 2013; 132 (1):8-17

5:Guskiewicz KM, McCrea M, Marshall SW, et al. Cumulative effects associated with recurrent concussion in collegiate football players: the NCAA Concussion Study. JAMA. 2003; 290(19):2549-2555.

6:Asken BM, Sullan MJ, DeKosky ST, et al. Research gaps and controversies in chronic traumatic encephalopathy: a review. JAMA Neurol. 2017; 74(10):1255-1262.

7:McCrory P, Johnston K, Meeuwisse W, et al. Summary and agreement statement of the 2nd International Conference on Concussion in Sport, Prague 2004. Br J Sports Med. 2005; 39(suppl 1):i78-i86.

8:Raftery M, Kemp S, Patricios J, et al. It is time to give concussion an operational definition: a 3-step process to diagnose (or rule out) concussion within $48 \mathrm{~h}$ of injury: World Rugby guideline. Br J Sports Med. 2016; 50(11): 642-643

9:http://playerwelfare.worldrugby.org/content/getfile.php?h=030cc0e5445023d28876281008 63ace2\&p=downloads/concussion/HIA_Protocol_Detailed_Description_Landscape_EN.pdf

10:McCrea M, Kelly JP, Randolph C, et al. Standardized assessment of concussion (SAC): onsite mental status evaluation of the athlete. J Head Trauma Rehabil. 1998; 13(2): 27-35.

11:McCrea, M. Standardized mental status testing on the sideline after sport-related concussion. J Athl Train. 2001; 36(3): 274-279

12:http://www.horsesportireland.ie/wp-content/uploads/2015/10/MEAI-ConcussionGuidelines-for-Equestrian-Sports1.pdf

13:Batterham AM, Hopkins WG. Making meaningful inferences about magnitudes. Int J Sports Physiol Perform. 2006; 1(1): 50-57.

14:Hopkins W, Marshall S, Batterham A. Progressive statistics for studies in sports medicine and exercise science. Med Sci Sports Exerc. 2009; 41(1): 3-13 
15:Lee JH, Howell DR, Meehan III WP, et al. Effects of Exercise on Sport Concussion Assessment Tool-Third Edition Performance in Professional Athletes. Orthop J Sports Med. 2017; 5(9).

16:Coutts A, Reaburn P, \& ABT, G. Hearth rate, blood lactate concetration and estimated energy expediture in a semi-profesional rugby league team during a match: case study. J Sports Sci. 2003; 21(2):97-103.

17:Wilkins JC, McLeod TCV, Perrin DH. Performance on the balance error scoring system decreases after fatigue. J Athl Train. 2004; 39(2):156-161.

18:Fox ZG, Mihalik JP, Blackburn JT, et al. Return of postural control to baseline after anaerobic and aerobic exercise protocols. J Athl Train. 2008; 43(5):456-463.

19:Schneiders AG, Sullivan SJ, Handcock P, et al. Sports concussion assessment: the effect of exercise on dynamic and static balance. Scand J Med Sci Sports. 2012; 22(1): 85-90.

20:Schneiders AG, Sullivan SJ, McCrory PR, et al. The effect of exercise on motor performance tasks used in the neurological assessment of sports-related concussion. Br J Sports Med. 2008; 42(12), 1011-1013.

21:McCrory P, Johnston K, Meeuwisse W, et al. Summary and agreement statement of the 2nd International Conference on Concussion in Sport, Prague 2004. Br J Sports Med. 2005; 39(suppl 1):i78-i86.

22:Chin EY, Nelson LD, Barr WB, et al. Reliability and validity of the Sport Concussion Assessment Tool-3 (SCAT3) in high school and collegiate athletes. Am J Sports Med. 2016. 44(9):2276-2285.

23:Downey RI, Hutchison MG, Comper P. (2018). Determining sensitivity and specificity of the Sport Concussion Assessment Tool 3 (SCAT3) components in university athletes. Brain Inj. 2018. 32(11):1-8.

24:Fuller GW, Govind O, Tucker R, et al. Sport concussion assessment tool-Third edition normative reference values for professional Rugby Union players. J Sci Med Sport. 2017; 21(4):47-51.

25: Yengo-Kahn AM, Hale AT, Zalneraitis BH, et al. (2016). The sport concussion assessment tool: a systematic review. Neurosurg focus. 2016; 40(4), E6.

26: Fuller GW, Kemp SPT, Raftery M. The accuracy and reproducibility of video assessment in the pitch-side management of concussion in elite rugby. J Sci Med Sport. 2017; 20(3):246249.

27: Hutchison MG, Comper P, Meeuwisse WH, et al. (2013). An observational method to code concussions in the National Hockey League (NHL): the heads-up checklist. Br J Sports Med. 2013;48(2):125-129. 
28: Moore RD, Lepine J, Ellemberg D. The independent influence of concussive and subconcussive impacts on soccer players' neurophysiological and neuropsychological function. Int J Psychophysiol. 2017;112: 22-30.

29: Hwang S, Ma L, Kawata K, et al. Vestibular dysfunction after subconcussive head impact. J Neurotrauma. 2017; 34(1): 8-15.

30: Dorminy M, Hoogeveen A, Tierney RT, et al. Effect of soccer heading ball speed on S100B, sideline concussion assessments and head impact kinematics. Brain inj. 2015; 29(10), 11581164.

31: Fuller CW, Fuller GW, Kemp SP, et al. Evaluation of World Rugby's concussion management process: results from Rugby World Cup 2015. Br J Sports Med. 2017; 51(1), 6469 


\section{Figure legends:}

Figure 1: Differences between baseline values and post effort in the different tests in the rugby union and regular sporting groups.

Figure 2: Between-group (rugby vs regular sporting) differences in the changes between baseline and post-effort values.

*: possibly; **: likely; ***: most likely; ****: almost certainly difference in the changes. Grey zone stands for trivial zone (effect size \pm 0.2 ). 